\title{
Comportamento alimentar de adolescentes em relação ao consumo de frutas e verduras
}

\author{
Adolescent eating behavior regarding fruit \\ and vegetable intakes
}

Natacha TORAL'

Betzabeth SLATER ${ }^{2}$

Isa de Pádua CINTRA ${ }^{3}$

Mauro FISBERG ${ }^{3}$

\section{R E S U M O}

\section{Objetivo}

Este estudo avaliou o comportamento alimentar pelo Modelo Transteorético e o estado nutricional de adolescentes de escolas de ensino técnico de São Paulo, quanto a seu consumo habitual de frutas e verduras.

\section{Métodos}

Investigaram-se o consumo alimentar habitual e a classificação nos estágios de mudança de comportamento, utilizando-se um questionário. Foram aferidos peso e altura para avaliação do estado nutricional pelo Índice de Massa Corporal. Para análise estatística, adotaram-se os testes "t" Student, Qui-Quadrado, Mann-Whitney e HSD-Tukey, com grau de significância de $5 \%$.

\section{Resultados}

Observou-se baixa prevalência de desvios nutricionais entre os 234 participantes: 3,8\% foram classificados como baixo peso e $12,4 \%$ apresentavam excesso de peso. Apenas 12,4\% e 10,3\% consumiam frutas e verduras, respectivamente, conforme o recomendado pela Pirâmide Alimentar. Cerca de um terço da amostra foi classificada tanto em pré-contemplação como em manutenção. Observou-se uma discrepância entre o consumo referido e a percepção alimentar, tendo em vista que $79,7 \%$ e $83,7 \%$ dos adolescentes acreditavam, erroneamente, que seu consumo de frutas e verduras, respectivamente, era saudável.

\section{Conclusão}

O alto percentual de adolescentes em pré-contemplação evidencia a importância do desenvolvimento de estratégias específicas contra a maior resistência desses a modificações dietéticas. A percepção errônea quanto às características de uma dieta saudável e o baixo consumo de frutas e verduras classificam os adolescentes

\footnotetext{
1 Mestranda, Faculdade de Saúde Pública, Universidade de São Paulo. Av. Dr. Arnaldo, 715, Cerqueira César, 01246-904, São Paulo, SP, Brasil. Correspondência para/Correspodence to: N. TORAL. E-mail: <natytb@usp.br>.

2 Departamento de Nutrição, Faculdade de Saúde Pública, Universidade de São Paulo. São Paulo, SP, Brasil.

3 Centro de Atendimento e Apoio ao Adolescente, Universidade Federal de São Paulo. São Paulo, SP, Brasil.
} 
como grupo de risco, exigindo atenção especial para a promoção de hábitos alimentares saudáveis e garantia de qualidade de vida.

Termos de indexação: adolescente; comportamento do adolescente; consumo de alimentos; estado nutricional.

\section{A B S T R A C T}

\section{Objective}

The purpose of this study was to evaluate the eating behavior using the Transtheoretical Model and the nutritional status of adolescents from technical schools of São Paulo regarding their usual fruit and vegetable intakes.

\section{Methods}

A questionnaire was used to collect data on the usual fruit and vegetable intakes and to classify the stages of change. Weight and height were measured to assess nutrional status using the body mass index. The statistical analysis comprised the Student's T-test, chi-square test, Mann-Whitney test and the HSD-Tukey test, all calculated using a significance level of $5 \%$.

\section{Results}

Low prevalence of nutritional disorders was observed among the 234 participants: $3.8 \%$ were classified as low weight and $12.4 \%$ were overweight. Only $12.4 \%$ and $10.3 \%$ had usual fruit and vegetable intakes, respectively, as recommended by the Food Guide Pyramid. Approximately one third of the sample was in the precontemplation and maintenance stages. A discrepancy was seen between self-rated food consumption and dietary perception, taking into account that $79.7 \%$ and $83.7 \%$ of the adolescents had a misperception about their fruit and vegetable intakes, respectively, considering them healthy.

\section{Conclusion}

The high prevalence of adolescents in the precontemplation stage shows the importance of developing specific strategies against their resistance to dietary changes. Their misperception related to the characteristics of a healthy diet and the low fruit and vegetable intakes classify adolescents as a risk group that needs special attention in order to promote healthy dietary habits and to guarantee quality of life.

Indexing terms: adolescent; adolescent behavior; food consumption; nutritional status.

\section{N T R O D U Ç Ã O}

As dificuldades para motivar os indivíduos a alterar o seu consumo alimentar têm sido muito estudadas, devendo-se considerar a complexa gama de fatores ambientais, nutricionais, psicológicos, sociais e culturais envolvidos nesse comportamento. Uma das maiores barreiras para a prática de mudanças na dieta é a crença de que não há necessidade de alteração dos hábitos alimentares, decorrente, na maioria das vezes, de uma interpretação errada do próprio consumo. Em diversos países foi observada alta prevalência de indivíduos que acreditam não ser necessário alterar sua dieta, por já possuírem uma alimentação saudável ${ }^{1-3}$. Contudo, restam dúvidas quanto aos reais meios de que a população dispõe para avaliar sua própria dieta, e se as supostas alterações dietéticas realizadas para a adoção de hábitos saudáveis correspondem às recomendações dos guias alimentares.

Esses dados representam um desafio para os nutricionistas em relação à busca de intervenções de sucesso, as quais sejam capazes de mobilizar os indivíduos para a adoção de práticas alimentares saudáveis. Um dos determinantes para que os indivíduos levem em consideração os comportamentos relacionados à saúde é a percepção da necessidade de praticar tal ação ${ }^{4}$. Pode-se inferir, portanto, que reconhecer a necessidade de alteração dos hábitos alimentares é um requisito fundamental para iniciar uma mudança dietética. 
A capacidade de avaliação individual dos aspectos dietéticos é um fator indispensável a ser considerado no delineamento de orientações para adolescentes. O consumo alimentar adotado nesse estágio de vida tem sérias implicações no crescimento, na promoção de saúde, a longo prazo, e no desenvolvimento do comportamento alimentar durante a vida adulta ${ }^{5}$. Porém, o estilo de vida nessa fase, ao sofrer diversas influências, como as exercidas pelo convívio familiar, amigos, mídia e pressão social, freqüentemente não proporcionam meios para o suprimento adequado das necessidades nutricionais, as quais estão aumentadas devido ao rápido crescimento e desenvolvimento ocorridos nesse período.

Como instrumento de auxílio à compreensão da mudança comportamental relacionada à saúde, diversos estudos têm aplicado o Modelo Transteorético de Mudança de Comportamento ${ }^{6,7}$. De acordo com esse modelo, as alterações no comportamento relacionado à saúde ocorrem por meio de cinco estágios distintos: pré-contemplação, contemplação, decisão, ação e manutenção ${ }^{6}$. No estágio pré-contemplativo, a mudança comportamental ainda não foi considerada pelo indivíduo, ou seja, não foram realizadas alterações no comportamento e não há intenção de adotá-las num futuro próximo. No estágio de contemplação, o indivíduo começa a considerar a mudança comportamental. Na decisão, o indivíduo já decidiu alterar seu comportamento no próximo mês, apesar de nenhuma mudança ter sido cumprida até o momento. O estágio de ação está relacionado ao indivíduo que alterou seu comportamento recentemente, nos últimos seis meses. No estágio de manutenção, o indivíduo já mudou seu comportamento e o manteve por mais de seis meses.

Foi observado que os indivíduos classificados nos estágios mais avançados constantemente apresentavam um consumo menor de gordura e maior de frutas, vegetais e fibras ${ }^{8}$. Nos Estados Unidos, constatou-se que adultos classificados no estágio de manutenção consumiam 0,99 e 0,68 porções diárias a mais de frutas e vegetais, respectivamente, quando comparados àqueles nos estágios de mudança alimentar anteriores ao da ação 9 .

Foi demonstrado que o desenvolvimento de intervenções específicas para cada estágio de mudança de comportamento alimentar é mais eficaz para a motivação dos indivíduos a adotar e manter o comportamento alterado ${ }^{10}$. Portanto, os programas de educação nutricional direcionados à população adolescente podem ser muito beneficiados se considerarem os diferentes estágios de mudança comportamental, tendo em vista que cada um deles corresponde a diferentes atitudes e percepções perante a nutrição e a saúde.

A proposta deste estudo foi avaliar o comportamento alimentar e o estado nutricional de alunos adolescentes de escolas profissionalizantes do Serviço Nacional de Aprendizagem Industrial (SENAI) de São Paulo, em relação ao seu consumo habitual de frutas e verduras.

\section{MÉ T O D O S}

Trata-se de um estudo observacional, de corte transversal. A amostra foi constituída por estudantes de duas escolas do (SENAI) de São Paulo, selecionadas por conveniência. A coleta de dados foi realizada no período de novembro a dezembro de 2003. Foram entrevistados, por meio de um questionário, todos os alunos voluntários que se encontravam na faixa etária correspondente à adolescência, isto é, de 10 a 19 anos ${ }^{11}$.

Foram coletadas as seguintes informações de cada participante: sexo, idade e dados antropométricos (peso e altura), seguindo procedimentos recomendados por Frisancho ${ }^{12}$. Tais dados foram utilizados para o cálculo do Índice de Massa Corporal (IMC), definido como a relação entre o peso em quilogramas e a altura em metros elevada ao quadrado $\left(\mathrm{kg} / \mathrm{m}^{2}\right)$. Este índice possibilitou a classificação do estado nutricional de acordo com a Organização Mundial da Saúde ${ }^{13}$, a partir da tabela de Must et al. ${ }^{14}$. 
O consumo de frutas e verduras foi avaliado, respectivamente, por meio das seguintes perguntas: "Com que freqüência você consome frutas ou suco de frutas natural" ? e "Com que freqüência você consome verduras e/ou legumes" ? O adolescente foi orientado a optar por uma das cinco alternativas: consumo diário, semanal, mensal, menos de uma vez por mês, raramente e nunca. Caso o consumo fosse diário, semanal ou mensal, o participante era questionado sobre quantas vezes por dia, semana ou mês consumia o citado alimento e, em seguida, quantas porções do mesmo eram habitualmente consumidas em cada vez. Para facilitar o entendimento dessa questão e a obtenção de sua resposta, o adolescente foi informado sobre o conceito de "porção" com o auxílio de um folheto explicativo, contendo alguns exemplos.

Em seguida, foi calculado o consumo médio de porções diárias de frutas, bem como o de verduras. No caso de consumo diário, foi feita a multiplicação do número de porções pelo número de vezes ao dia em que as mesmas eram consumidas. Para o semanal, foi feita a multiplicação entre o número de vezes por semana e o número de porções consumidas por vez, dividindose, então, o valor obtido por 7. Caso fosse referido consumo mensal desses alimentos, o resultado da multiplicação entre o número de vezes por mês e o número de porções consumidas em cada ocasião era dividido por 30 .

O consumo médio de porções diárias, assim obtido, foi comparado às recomendações de consumo de frutas e verduras sugeridas pela Pirâmide Alimentar proposta por Philippi et al. ${ }^{15}$. Sugere-se como adequado o consumo de 4 a 5 porções diárias de verduras e de 3 a 5 porções diárias de frutas. Por questões metodológicas, foi considerada uma casa decimal para o número de porções consumidas: estabeleceu-se que a faixa adequada em relação a frutas seria de 2,5 a 5,4 porções e para as verduras, de 3,5 a 5,4 porções.

Além da comparação com os parâmetros da Pirâmide Alimentar, adotou-se um segundo critério para avaliar a adequação do consumo de frutas e verduras. Para aumentar a capacidade de identificação de grupos expostos a maior risco de inadequação de consumo, foi estabelecida, como ponto de investigação, a prevalência de indivíduos que consumiam menos de 1,0 porção ao dia, para ambos os grupos alimentares. Os adolescentes assim classificados estariam muito aquém das recomendações propostas pela Pirâmide, constituindo um importante grupo em zona de vigilância para inadequação.

Foram excluídos os adolescentes que referiram consumo superior a 15 porções diárias $(n=3)$, quando somadas frutas e verduras.

Além disso, cada adolescente foi questionado a respeito de seu comportamento alimentar em relação ao consumo de frutas e verduras, de forma a classificá-lo nos cinco estágios de mudança: pré-contemplação, contemplação, decisão, ação, manutenção ${ }^{6}$. Tal classificação foi realizada de forma independente para cada um dos grupos alimentares estudados, por meio de um algoritmo (Anexo) adaptado de publicações prévias $^{8,16}$.

Avaliou-se a aderência das variáveis quantitativas à distribuição normal, utilizando-se o teste Kolmogorov-Smirnov. Teste paramétrico ( " $t$ " Student) foi utilizado para as análises que envolveram a variável idade e testes não-paramétricos (Qui-Quadrado e Mann-Whitney) para as que incluíram consumo alimentar de frutas e verduras. Utilizou-se o teste HSD-Tukey (Tukey Honest Significant difference test) para análise de comparações múltiplas do consumo alimentar entre os estágios de mudança de comportamento. A análise estatística foi realizada com o auxílio do programa de computador Statistical Package for the Social Sciences ${ }^{17}$, versão 10.0, sendo adotado o grau de significância de 5\% $(p<0,05)$.

A realização deste trabalho foi analisada e aprovada pelo Comitê de Ética em Pesquisa da Universidade Federal de São Paulo (protocolo n'1374/03). 


\section{RESULTADOS}

A amostra foi constituída por 234 alunos do Senai, sendo $21,8 \%$ do sexo feminino e $78,2 \%$ do sexo masculino, com média (e desvio-padrão) de idade de 16,16 $(0,7)$ anos.

Em relação ao estado nutricional, verificou-se baixa prevalência de desvios nutricionais entre os adolescentes do estudo, sendo que não foram encontradas diferenças significativas entre os sexos quanto ao estado nutricional $(p>0,05)$. Observou-se que 3,8\% da amostra apresentava baixo peso, enquanto que o excesso de peso foi encontrado em menos de $13,0 \%$ da amostra: $9,8 \%$ e $2,6 \%$ dos adolescentes apresentavam sobrepeso e obesidade, respectivamente.

A mediana de consumo dos grupos alimentares estudados entre os adolescentes foi de 0,97 e 1,2 porções diárias para frutas e verduras, respectivamente. Observou-se diferença significativa $(p=0,020)$ entre o consumo de frutas de acordo com o sexo (Tabela 1). O mesmo não foi observado em relação ao consumo de verduras $(p=0,124)$, apesar das meninas terem apresentado consumo maior desses alimentos.

Em relação ao consumo recomendado pela Pirâmide Alimentar, foi observado consumo inferior ao proposto, tanto para frutas como para verduras. Isso foi observado em aproximadamente $89 \%$ dos

Tabela 1. Estatística descritiva do consumo de frutas e verduras (resultados em porções diárias), segundo sexo dos adolescentes entrevistados. Escolas do SENAI, São Paulo, 2003.

\begin{tabular}{lccccc}
\hline Variável & $\mathrm{n}$ & \multicolumn{4}{c}{ Mediana Mínimo Máximo $p$-valor } \\
\hline Consumo de frutas & & & & & \\
Meninos & 183 & 1,00 & 0 & 12 & \\
Meninas & 51 & 0,86 & 0 & 4 & \\
Total & 234 & 0,97 & 0 & 12 & $0,020^{*}$ \\
Consumo de verduras & & & & \\
Meninos & 183 & 1,10 & 0 & 12 & \\
Meninas & 51 & 1,70 & 0 & 12 & \\
Total & 234 & 1,20 & 0 & 12 & $0,124^{*}$ \\
\hline
\end{tabular}

*Comparação de médias de consumo entre os sexos pelo teste de Mann-Whitney. alunos avaliados e não houve diferenças estatisticamente significativas entre os sexos (Figura 1). Entre esses, verificou-se que o consumo inferior a uma porção diária representava 50,0\% da amostra total, em relação ao consumo de frutas, e 38,9\% para verduras.

A classificação dos adolescentes, segundo o estágio de mudança de comportamento alimentar, revelou que cerca de um terço da amostra encontrava-se em pré-contemplação e outro terço em manutenção (Figura 2). Não foram observadas diferenças entre estágios de mudança e o sexo ou estado nutricional dos participantes $(p>0,05)$. Por outro lado, constatou-se a associação de tais estágios e o consumo de frutas e verduras (Tabela 2).

A comparação do percentual de indivíduos classificados no estágio de manutenção (29,5\% e $38,9 \%$ para o consumo de frutas e verduras, respectivamente) com a constatação de que $12,4 \%$ e $10,3 \%$ dos adolescentes entrevistados apresentaram consumo adequado de frutas e verduras, respectivamente, incitou uma avaliação da freqüência de respostas à primeira pergunta do algoritmo utilizado para a classificação nos diferentes estágios de mudança de comportamento. Observou-se que, dos 79 adolescentes que acreditavam ter consumo saudável de frutas,

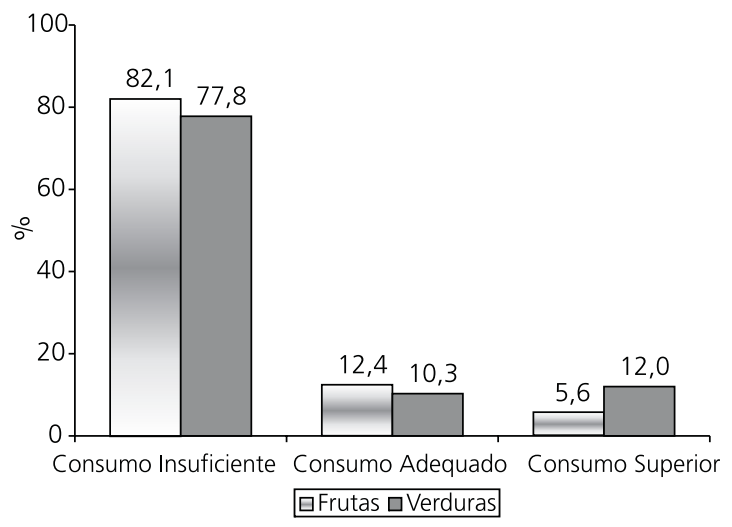

Figura 1. Distribuição percentual da adequação do consumo de frutas e verduras em relação às recomendações da Pirâmide Alimentar entre os adolescentes entrevistados. Escolas do SENAl, São Paulo, 2003. 


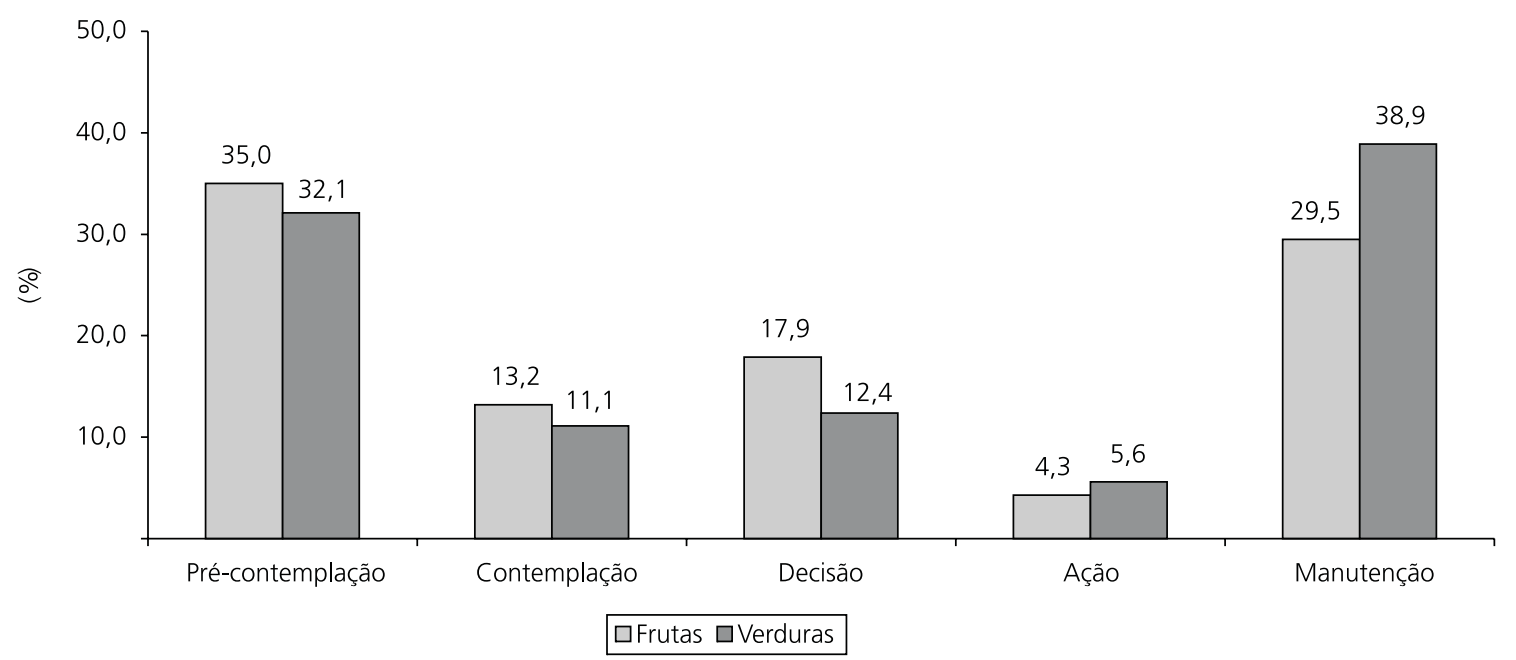

Figura 2. Distribuição percentual dos adolescentes entrevistados, de acordo com os estágios de mudança de comportamento alimentar em relação ao consumo de frutas e verduras. Escolas do SENAl, São Paulo, 2003.

Tabela 2. Comparação do consumo médio diário de frutas e verduras em porções ingeridas de acordo com os estágios de mudança de comportamento alimentar dos adolescentes entrevistados (resultados apresentados como média desvio-padrão)*. Escolas do SENAI, São Paulo, 2003.

\begin{tabular}{|c|c|c|c|c|c|}
\hline \multirow{3}{*}{$\begin{array}{l}\text { Estágio de mudança } \\
\text { de comportamento } \\
\text { Pré-contemplação }\end{array}$} & \multicolumn{5}{|c|}{ Consumo médio (porção/dia) } \\
\hline & \multicolumn{2}{|r|}{ Frutas } & \multicolumn{3}{|c|}{ Verduras } \\
\hline & 0,9 & $\pm 1,3^{\mathrm{a}}$ & 0,8 & \pm & $1,0^{\mathrm{a}, \mathrm{c}}$ \\
\hline Contemplação & 1,1 & $\pm 1,3^{\mathbf{b}}$ & 1,1 & \pm & $1,1^{\mathrm{a}, \mathrm{c}}$ \\
\hline Decisão & 1,5 & $\pm 1,6$ & 1,7 & \pm & $1,7^{\mathrm{a}, \mathrm{c}}$ \\
\hline Ação & 2,0 & $\pm 1,6$ & 4,7 & \pm & 3,3 \\
\hline Manutenção & 2,2 & $\pm 2,0$ & 4,2 & \pm & 4,4 \\
\hline
\end{tabular}

*Comparação de médias de consumo entre os estágios pelo teste HSD-Tukey; ${ }^{\text {a: }}$ diferença significativa em relação ao estágio de manutenção $(p \leq 0,001)$; $\mathbf{b}$ : diferença significativa em relação ao estágio de

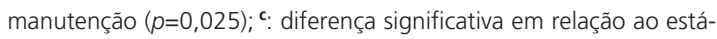
gio de ação $(p \leq 0,001)$.

somente 16, de fato, consumiam esses alimentos de forma adequada. A mesma análise, para o consumo de verduras, evidenciou que, dos 104 adolescentes que afirmaram consumir esses alimentos de forma saudável, 17 indivíduos estavam verdadeiramente corretos. Dessa forma, verificou-se que $79,7 \%$ e $83,7 \%$ da amostra estava errada ao acreditar que possuía hábitos alimentares adequados em relação ao consumo de frutas e verduras, respectivamente.
D I S C U S S Ã O

Trata-se de um estudo realizado em escolas profissionalizantes caracterizadas pelo percentual elevado de alunos do sexo masculino, o que pode decorrer da preferência destes pelos cursos oferecidos, como automobilística e eletroeletrônica.

Entre os adolescentes avaliados, verificou-se uma taxa de excesso de peso inferior à encontrada em outros estudos realizados no Brasil, que apontam para uma prevalência de mais de $20 \%$ nesse estágio de vida ${ }^{18,19}$. Pesquisas de base populacional destacam um aumento alarmante das taxas de excesso de peso na adolescência ${ }^{20}$. Dados do Estudo Nacional de Despesa Familiar (ENDEF), realizado em 1974-75, indicavam que 2,6\% dos meninos e 5,8\% das meninas apresentavam excesso de peso. Tais percentuais atingiram $11,8 \%$ e $15,3 \%$, respectivamente, segundo a Pesquisa sobre Padrões de Vida (PPV), de 1996-1997.

Esse quadro epidemiológico é atribuído ao sedentarismo e à adoção de práticas alimentares inadequadas na adolescência. É freqüente o consumo excessivo de refrigerantes, açúcares e fast food, além da baixa ingestão de frutas e verduras na adolescência ${ }^{21-23}$. Contudo, a baixa prevalência de obesidade, encontrada neste 
estudo, pode ser atribuída a um estilo de vida diferenciado de grande parte dos alunos avaliados, os quais realizam uma jornada tripla, que inclui um turno na escola, um turno no SENAI e um turno de trabalho. Tal característica difere de muitos adolescentes, que se restringem às atividades escolares e dispõem de longos períodos de ociosidade.

O baixo consumo de frutas e verduras relatado na literatura, tanto entre adolescentes como na população adulta, é semelhante ao encontrado neste estudo. Glanz et al. ${ }^{8}$ e Stables et al. ${ }^{24}$ observaram que o número de porções de frutas e verduras consumidas diariamente entre adultos americanos aumentou de 2,10, em 1990, para 3,98, em 1997. Tal melhora tem sido atribuída à implementação do programa 5-A-Day for a Better Health, em 1991, uma campanha de educação nutricional, cuja finalidade é aumentar a conscientização quanto à importância do consumo adequado de frutas e vegetais nos EUA, representado por, no mínimo, 5 porções diárias.

O consumo de menos de uma porção diária de frutas e verduras foi próximo aos valores encontrados pelo Minnesota Adolescent Health Survey em 1996, o qual mostrou que cerca de $40,0 \%$ dos adolescentes americanos apresentava esse hábito ${ }^{25}$. Da mesma forma, observou-se, neste estudo, que o percentual de adolescentes que apresentaram consumo igual ou superior a cinco porções diárias de frutas e verduras (somadas) foi de 28,3\% (dados não apresentados), enquanto que nos Estados Unidos, o Youth Risk Behavior Surveillance, realizado em 2001, identificou que apenas $21,4 \%$ dos jovens avaliados havia consumido tal quantidade no dia anterior à entrevista ${ }^{26}$.

Há controvérsias na literatura quanto à melhor adequação de consumo de frutas e verduras entre os sexos: Grunbaum et al. ${ }^{26}$ atribuem essa prática aos homens, enquanto que Stables et al. ${ }^{24}$ e Neumark-Sztainer et al. ${ }^{25}$ afirmam o mesmo para as mulheres. Neste estudo, os meninos apresentaram maior consumo de frutas em comparação com as meninas. Isso pode ser atribuído ao fato de que 7,0\% dos adolescentes do sexo masculino apresentavam consumo de frutas superior ao recomendado (5,5 porções/dia ou mais), sendo que nenhuma menina encontrava-se nessa condição.

A alta prevalência de indivíduos no estágio de pré-contemplação indica a importância do desenvolvimento de estratégias específicas para os mesmos, tendo em vista que são caracterizados por mostrar maior resistência frente a possíveis mudanças na dieta. Deve-se ter em mente que motivá-los exigirá grande dedicação dos profissionais de saúde, os quais deverão focalizar os programas de educação nutricional no fornecimento de informações básicas sobre hábitos alimentares adequados, aumentando o conhecimento sobre seus benefícios e destacando sua relevância para a saúde ${ }^{16}$. Logo, sugere-se que sejam planejadas intervenções específicas, diferenciadas segundo o grau de motivação, para a prática de mudanças na dieta.

A associação entre os estágios de mudança de comportamento e o consumo de frutas e verduras está de acordo com o descrito em diversos estudos $^{8,27,28}$. Esse fato foi mais significativo, quando comparado o consumo observado em pré-contemplação e manutenção, evidenciando que os estágios mais avançados mostram maior consumo de frutas e verduras e representam comportamentos alimentares distintos em relação aos estágios iniciais.

O fato de, aproximadamente, um terço dos adolescentes terem sido classificados no estágio mais avançado (manutenção) contrastou com o baixo percentual de consumo adequado de frutas e verduras. A análise detalhada das respostas ao algoritmo de classificação nos estágios de mudança de comportamento, quando comparadas ao consumo alimentar referido de frutas e verduras, possibilitou a identificação de uma percepção errônea dos aspectos salutares da dieta entre os adolescentes. Assim como observado neste estudo, Kristal et al. ${ }^{16}$ afirmam que outros autores constataram discrepância entre as classificações nos diferentes estágios, realizadas por intermédio 
da avaliação do consumo alimentar, em comparação com os resultados obtidos a partir da percepção do indivíduo sobre sua própria dieta.

\section{O N CLUSÃ O}

Evidenciou-se a importância da avaliação do consumo alimentar em associação com o Modelo Transteorético, possibilitando a identificação de discrepâncias entre a percepção dos aspectos referentes à própria dieta e a adoção de hábitos em concordância com as recomendações dietéticas. Dessa forma, é possível eliminar a ambigüidade existente no auto-relato do hábito alimentar. A associação observada entre o consumo de frutas e verduras e os estágios de mudança de comportamento justifica a utilização do modelo, além de ter evidenciado dados fundamentais para a melhor compreensão do comportamento alimentar de adolescentes.

Esses dados mostram que os participantes deste estudo não reconhecem suas práticas alimentares inadequadas, o que representa um obstáculo aos programas de educação nutricional, tendo em vista que esses indivíduos não estarão motivados a realizar alterações dietéticas. Tal constatação, associada ao baixo consumo de frutas e verduras, observado na amostra, possibilita a classificação dos adolescentes como um grupo de risco, que exige atenção especial para a promoção de hábitos alimentares saudáveis e garantia de qualidade de vida futura.

\section{REFERÊ NCIAS}

1. Reid DJ, Conrad SA, Hendricks SM. Tracking nutrition trends, 1989-1994: an update on Canadians' attitudes, knowledge and reported actions. Can J Public Health. 1996; 87(2):113-8.

2. Kearney M, Gibney MJ, Martinez JA, Almeida MDV, Friebe D, Zunft HJF, et al. Perceived need to alter eating habits among representative samples of adults from all member states of the European Union. Eur J Clin Nutr. 1997; 51(Suppl 2):S30-S5.

3. Biloukha $\mathrm{O}$, Utermohlen $\mathrm{V}$. Healthy eating in Ukraine: attitudes, barriers and information sources. Public Health Nutr. 2001; 4(2):207-15.
4. Janz NK, Becker MH. The Health Belief Model: a decade later. Health Educ Q. 1984; 11(1):1-47.

5. Neumark-Sztainer D, Story M, Perry C, Casey MA. Factors influencing food choices of adolescents: findings from focus-groups discussions with adolescents. J Am Diet Assoc. 1999; 99(8): 929-34, 7.

6. Prochaska JO, Di Clemente CC, Norcross JC. In search of how people change: applications to addictive behaviors. Am Psychol. 1992; 47(9): 1102-14.

7. Ni-Mhurchu C, Margetts BM, Speller VM. Applying the stages-of-change model to dietary change. Nutr Rev. 1997; 55(1 Pt 1):10-6.

8. Glanz K, Kristal AR, Heimendinger J, Linnan L McLerran DF. Stages of change in adopting healthy diets: fat, fiber, and correlates of nutrient intake. Health Educ Q. 1994; 21(4):499-519.

9. Trudeau E, Kristal AR, Li S, Patterson RE. Demographic and psychosocial predictors of fruit and vegetable intakes differ: implications for dietary interventions. J Am Diet Assoc. 1998; 98(12):1412-7.

10. Brug J, Glanz K, van Assema P, Kok G, van Breukelen GJ. The impact of computer-tailored feedback and iterative feedback on fat, fruit, and vegetable intake. Health Educ Behav. 1998; 25(4):517-31.

11. Organización Mundial de la Salud. La salud de los jóvenes: un reto y una esperanza. Ginebra; 1995.

12. Frisancho AR. Anthropometric standards for the assessment of growth and nutritional status. Ann Arbor, Michigan: Univesity of Michigan Press; 1990.

13. World Health Organization. Physical status: the use and interpretation of antropometry. Geneva; 1995. WHO Technical Report Series, n. 854.

14. Must A, Dallal GE, Dietz WH. Reference data for obesity: 85th and 95th percentiles of body mass index (wt/ht2): a correction. Am J Clin Nutr. 1991; 53(4):839-46.

15. Philippi ST, Latterza AR, Cruz ATR, Ribeiro LC. Pirâmide alimentar adaptada: guia para escolha dos alimentos. Rev Nutr. 1999; 12(1):65-80.

16. Kristal AR, Glanz K, Curry SJ, Patterson RE. How can stages of change be best used in dietary interventions? J Am Diet Assoc. 1999; 99(6): 679-84.

17. Statistical Package for the Social Sciences for Windows Student Version/ SPSS. Release 10.0 Chicago: Marketing Departament; 2000.

18. Fonseca VM, Sichieri R, Veiga GV. Fatores associados à obesidade em adolescentes. Rev Saúde Pública. 1998; 32(6):541-9. 
19. Monteiro POA, Victora CG, Barros FC, Tomasi E. Diagnóstico de sobrepeso em adolescentes: estudo do desempenho de diferentes critérios para o Índice de Massa Corporal. Rev Saúde Pública. 2000; 34(5):506-13.

20. Veiga GV, Cunha AS, Sichieri R. Trends in overweight among adolescents living in the poorest and richest regions of Brazil. Am J Public Health. 2004; 94(9):1544-8.

21. Fisberg M, Bandeira CRS, Bonilha EA, Halpern G, Hirschbruch MD. Hábitos alimentares na adolescência. Pediatr Mod. 2000; 36(11):724-34.

22. Pedrinola F. Nutrição e transtornos alimentares na adolescência. Pediatr Mod. 2002; 38(8):377-80.

23. Fisberg M. Atualização em obesidade na infância e adolescência. São Paulo: Atheneu; 2004.

24. Stables GJ, Subar AF, Patterson BH, Dodd K, Heimendinger J, Duyn MAV, et al. Changes in vegetable and fruit consumption and awareness among US adults: results of the 1991 and 19975 A day for better health program surveys. J Am Diet Assoc. 2002; 102(6):809-17.
25. Neumark-Sztainer D, Story M, Resnick MD, Blum RW. Correlates of inadequate fruit and vegetable consumption among adolescents. Prev Med. 1996; 25(5):497-505.

26. Grunbaum JO, Kann L, Kinchen SA, Williams B, Ross JG, Lowry $R$, et al. Youth risk behavior surveillance- united states, 2001. CDC Surveillance Summaries. Morb Mortal Wkly Rep. 2002; 51(SS-4):1-68.

27. Laforge RG, Greene GW, Prochaska JO. Psychosocial factors influencing low fruit and vegetable consumption. J Behav Med. 1994; 17(4):361-74.

28. Campbell MK, Symons M, Demark-Wahnefried W, Polhamus B, Bernhardt JM, McClelland JW, et al. Stages of change and psychosocial correlates of fruit and vegetable consumption among rural african-american church members. Am J Health Promot. 1998; 12(3):185-91.

Recebido em: 21/12/2004

Versão final reapresentada em: 10/8/2005

Aprovado em: 26/8/2005 


\section{ANEXO \\ ALGORITMO UTILIZADO PARA A CLASSIFICAÇÃO DOS INDIVÍDUOS NOS ESTÁGIOS DE MUDANÇA \\ DE COMPORTAMENTO EM RELAÇÃO AO CONSUMO DE FRUTAS E VERDURAS}

\section{Comportamento alimentar: consumo de frutas}

1. Você acha que seu consumo de frutas, atualmente, pode ser considerado adequado ou saudável?
(1) Sim (próxima questão)
(2) Não (questão 3)

2. Há quanto tempo você acha que seu consumo de frutas pode ser considerado adequado ou saudável?
(1) Menos de 1 mês / Entre 1 e 5 meses (AÇÃO)
(2) Entre 6 e 11 meses / Há 1 ano ou mais (MANUTENÇÃO)

3. Você tem pensado seriamente em aumentar seu consumo de frutas nos próximos meses?
(1) Sim (próxima questão)
(2) Não (PRÉ-CONTEMPLAÇÃO)

4. Como está o seu grau de confiança quando você pensa que aumentará seu consumo de frutas em breve (nos próximos dias ou no próximo mês)?

(1) Confiante ou muito confiante (DECISÃO/ PREPARAÇÃO)

(2) Pouco confiante ou não muito confiante (CONTEMPLAÇÃO)

\section{Comportamento alimentar: consumo de verduras}

1. Você acha que seu consumo de verduras e legumes, atualmente, pode ser considerado adequado ou saudável?
(1) $\operatorname{Sim}$ (próxima questão)
(2) Não (questão 3)

2. Há quanto tempo você acha que seu consumo de verduras e legumes pode ser considerado adequado ou saudável?

(1) Menos de 1 mês / Entre 1 e 5 meses (AÇÃO)

(2) Entre 6 e 11 meses / Há 1 ano ou mais (MANUTENÇÃO)

3. Você tem pensado seriamente em aumentar seu consumo de verduras e legumes nos próximos meses?
(1) Sim (próxima questão)
(2) Não (PRÉ-CONTEMPLAÇÃO)

4. Como está o seu grau de confiança quando você pensa que aumentará seu consumo de verduras e legumes em breve (nos próximos dias ou no próximo mês)?

(1) Confiante ou muito confiante (DECISÃO/ PREPARAÇÃO)

(2) Pouco confiante ou não muito confiante (CONTEMPLAÇÃO) 